\title{
ASYMPTOTIC PROPERTIES OF KOLMOGOROV WIDTHS
}

\author{
MIKHAIL I. OSTROVSKII
}

(Received 9 June 2009)

\begin{abstract}
We consider two problems concerning Kolmogorov widths of compacts in Banach spaces. The first problem is devoted to relations between the asymptotic behavior of the sequence of $n$-widths of a compact and of its projections onto a subspace of codimension one. The second problem is devoted to comparison of the sequence of $n$-widths of a compact in a Banach space $\mathcal{Y}$ and of the sequence of $n$-widths of the same compact in other Banach spaces containing $\mathcal{Y}$ as a subspace.
\end{abstract}

2000 Mathematics subject classification: primary 46B20; secondary 41A46, 41A65.

Keywords and phrases: absolute widths, Banach space, Kolmogorov widths.

\section{Introduction}

Our Banach space theory terminology and notation follow [3]. We denote the closed unit ball of a Banach space $\mathcal{Y}$ by $B \mathcal{Y}$, the unit ball of $\ell_{p}^{n}$ by $B_{p}^{n}$, and the norm closure of a set $M \subset \mathcal{Y}$ by $\bar{M}$. Let $\mathcal{Z}$ be a subset of a Banach space $\mathcal{X}$ and $x \in \mathcal{X}$. The distance from $x$ to $\mathcal{Z}$ is defined as $\operatorname{dist}(x, \mathcal{Z})=\inf \{\|x-z\|: z \in \mathcal{Z}\}$.

Definition 1.1. Let $K$ be a subset of a Banach space $\mathcal{X}, n \in \mathbb{N} \cup\{0\}$. The Kolmogorov $n$-width of $K$ is given by

$$
d_{n}(K, \mathcal{X})=\inf _{\mathcal{X}_{n}} \sup _{x \in K} \operatorname{dist}\left(x, \mathcal{X}_{n}\right),
$$

where the infimum is over all $n$-dimensional subspaces. We use $d_{n}(K)$ instead of $d_{n}(K, \mathcal{X})$ if $\mathcal{X}$ is clear from context.

This notion was introduced by Kolmogorov [6] in 1936. It has been the subject of extensive study and has found many applications. See [7, 11, 14] for information on the Kolmogorov $n$-width. In [9] it was discovered that some general asymptotic properties of Kolmogorov widths are useful in the study of closures of sets of operators in the weak operator topology. The purpose of this paper is to continue the analysis of asymptotic properties of widths.

Supported by St. John's University Summer 2009 Support of Research Program.

(C) 2010 Australian Mathematical Publishing Association Inc. 0004-9727/2010 \$16.00 
One of the results on asymptotic properties of widths proved in [9, Lemma 3.3] is the following lemma.

LEMMA 1.2. Let $K$ be a bounded subset in a Banach space $\mathcal{X}$. If $K_{0}=K \cap L$, where $L$ is a closed linear subspace in $\mathcal{X}$ which does not contain $K$, then there exists a constant $0<C<\infty$ such that $d_{n}\left(K_{0}\right) \leq C d_{n+1}(K)$ for all $n \in \mathbb{N} \cup\{0\}$.

In view of possible applications in the spirit of [9] it is important to find out whether one can prove a version of Lemma 1.2 for projections. In this connection we consider the following problem. Let $K$ be a compact in a Banach space $\mathcal{X}$ such that $\mathcal{X}=\varlimsup \operatorname{lin}(K)$. Let $K_{0}=P(K)$, where $P$ is a bounded linear projection of $\mathcal{X}$ onto its subspace of codimension one. Does there exist $0<C<\infty$ such that $d_{n}\left(K_{0}, \mathcal{X}\right) \leq C d_{n+1}(K, \mathcal{X})$ for all $n \in \mathbb{N} \cup\{0\}$ ? The first purpose of this paper is to answer this problem in the negative. Our example is an infinite-dimensional ellipsoid in a Hilbert space.

Definition 1.3. A set $K$ of the form $A\left(B_{\mathcal{H}_{0}}\right)$, where $A$ is an infinite-dimensional bounded compact operator from a Hilbert space $\mathcal{H}_{0}$ to a Hilbert space $\mathcal{H}$, is called an ellipsoid. An ellipsoid $K$ is called lacunary if

$$
\liminf _{n \rightarrow \infty}\left(d_{n+1}(K, \mathcal{H}) / d_{n}(K, \mathcal{H})\right)=0
$$

THEOREM 1.4. There exists a lacunary ellipsoid $K$ with dense linear span in $a$ Hilbert space $\mathcal{H}$ and an orthogonal projection $P: \mathcal{H} \rightarrow \mathcal{H}$ with one-dimensional kernel such that there exists $0<C<\infty$ for which $d_{n}(K, \mathcal{H}) \leq C d_{n}(P(K), \mathcal{H})$.

Since for a lacunary ellipsoid $K$ there is no $0<C<\infty$ such that $d_{n}(K, \mathcal{H}) \leq$ $C d_{n+1}(K, \mathcal{H})$, Theorem 1.4 answers the above-mentioned problem in the negative. Theorem 1.4 is proved in Section 2.

Another problem considered in this paper is related to the well-known observation that there exist a Banach space $\mathcal{X}$, its closed subspace $\mathcal{Y}$, and a subset $K \subset \mathcal{Y}$ such that for some $n$ the strict inequality

$$
d_{n}(K, \mathcal{X})<d_{n}(K, \mathcal{Y})
$$

holds. Examples of this type can be found in [1], [7, p. 446, Problem 10.3], [11, pp. 10 and 35], and [14]. Observe that the nonstrict inequality in (1.1) follows immediately from the definition and holds for all triples $K \subset \mathcal{Y} \subset \mathcal{X}$.

Let $K$ be a compact in a Banach space $\mathcal{X}$ and let $\mathcal{Y}=\varlimsup$ in $K$. We consider the following problem: how small in comparison with $d_{n}(K, \mathcal{Y})$ can $d_{n}(K, \mathcal{X})$ be? Using Kashin's decomposition (see [4, 13]) we obtain the following result (proved in Section 3).

THEOREM 1.5. For each $n$ the Banach space $\ell_{1}^{3 n}$ contains a $2 n$-dimensional subspace $\mathcal{Y}_{2 n}$ and a compact $K_{n} \subset \mathcal{Y}_{2 n}$ such that $d_{n}\left(K_{n}, \ell_{1}^{3 n}\right) \leq 1$, but $d_{n}\left(K_{n}, \mathcal{Y}_{2 n}\right) \geq c \sqrt{n}$ for some absolute constant $c>0$. 
Theorem 1.5 shows that the sequence $\left\{d_{n}(K, \mathcal{X})\right\}_{n}$ depends heavily on the ambient Banach space. In this connection it is natural to recall a notion introduced in [2, Section 2].

Definition 1.6. Let $K$ be a compact in a Banach space $\mathcal{Y}$ and $n \in \mathbb{N}$. The $n$th absolute width $d_{n}^{a}(K)$ of $K$ is defined by $d_{n}^{a}(K)=\inf _{\mathcal{X}} d_{n}(K, \mathcal{X})$, where the inf is over all Banach spaces $\mathcal{X}$ containing $\mathcal{Y}$ as a subspace.

In Section 4 we use Theorem 1.5 to establish one asymptotic property of $d_{n}^{a}$.

It is worth mentioning that some aspects of the natural problem of evaluating or estimating numbers $\left\{d_{n}^{a}(K)\right\}$, given a compact $K$ in a Banach space $\mathcal{Y}$, were considered in $[5,8]$.

\section{Asymptotic properties of widths of quotients}

Proof of Theorem 1.4. Let $\left\{e_{i}\right\}$ be the unit vector basis in $\mathcal{H},\left\{\beta_{i}\right\}$ be a sequence satisfying $\beta_{i} \geq \beta_{i+1}>0$, and let

$$
K=\left\{\sum_{i=1}^{\infty} x_{i} e_{i}: \sum_{i=1}^{\infty}\left(\frac{x_{i}}{\beta_{i}}\right)^{2} \leq 1\right\}
$$

Let $v=\sum_{i=1}^{\infty} a_{i} e_{i}$ be a unit vector $(\|v\|=1)$ and let $P$ be an orthogonal projection in $\mathcal{H}$ whose kernel is the linear span of $v$, so $P x=x-\langle x, v\rangle v$. Desired properties of sequences $\left\{\beta_{i}\right\}$ and $\left\{a_{i}\right\}$ will be described later. It is well known that $d_{n}(K)=\beta_{n+1}$ (see [7, p. 401]).

The well-known results on widths (see [7, Ch. 13, Section 5]) imply that in order to prove the theorem it suffices to show that for suitable $\left\{\beta_{n}\right\},\left\{a_{n}\right\}$, and $c>0$ we have $\inf \left\{\|P x\|: x \in \mathcal{S}_{n}\right\} \geq c \beta_{n}$, where

$$
\mathcal{S}_{n}=\left\{\sum_{i=1}^{\infty} x_{i} e_{i}: \sum_{i=1}^{n}\left(\frac{x_{i}}{\beta_{i}}\right)^{2}=1 \text { and } 0=x_{n+1}=x_{n+2}=\cdots\right\} .
$$

Since $\beta_{n+1}=d_{n}(K)$ and we are looking for a lacunary ellipsoid, we require that $\left\{\beta_{n}\right\}$ satisfies $\liminf _{n \rightarrow \infty}\left(\beta_{n+1} / \beta_{n}\right)=0$.

To simplify the computation, we prove that inf $\left\{\|P x\|: x \in \mathcal{R}_{n}\right\} \geq c \beta_{n}$ for

$$
\mathcal{R}_{n}=\left\{\sum_{i=1}^{\infty} x_{i} e_{i}: \frac{\sum_{i=1}^{n-1} x_{i}^{2}}{\beta_{n-1}^{2}}+\left(\frac{x_{n}}{\beta_{n}}\right)^{2}=1 \text { and } 0=x_{n+1}=x_{n+2}=\cdots\right\} .
$$

Let $\left\{x_{i}\right\}_{i=1}^{\infty} \in \mathcal{R}_{n}$. Denote by $v(n)$ the orthogonal projection of $v$ onto the subspace $E_{n}$ spanned by the first $n$ vectors of $\left\{e_{i}\right\}$. Then

$$
\|P x\|^{2}=\|x\|^{2}-\langle x, v\rangle^{2} .
$$


Let $x=w+z e_{n} \in \mathcal{R}_{n}$, where $w$ is the orthogonal projection of $x$ to $E_{n-1}$. Then $\|x\|^{2}=\|w\|^{2}+z^{2}$. Also

$$
\left(\frac{\|w\|}{\beta_{n-1}}\right)^{2}+\left(\frac{z}{\beta_{n}}\right)^{2}=1
$$

and $\langle x, v\rangle=\langle w, v(n-1)\rangle+z a_{n}$. Therefore

$$
\begin{aligned}
\|P x\|^{2} & =\|w\|^{2}+z^{2}-\left(\langle w, v(n-1)\rangle+z a_{n}\right)^{2} \\
& \geq\|w\|^{2}+z^{2}-\left(\|w\|\|v(n-1)\|+z a_{n}\right)^{2} \\
& =\|w\|^{2}\left(1-\|v(n-1)\|^{2}\right)+z^{2}\left(1-a_{n}^{2}\right)-2\|w\|\|v(n-1)\| z a_{n} .
\end{aligned}
$$

We denote the first two summands in the last line by $B^{2}$ and $C^{2}$, respectively. We show that for a suitable choice of the sequence $\left\{a_{n}\right\}$ there is a constant $0<d<1$ which does not depend on $n$ and is such that

$$
2\|w\|\|v(n-1)\| z a_{n} \leq 2 d B C
$$

Assume that we have shown (2.2). Then we get

$$
\|P x\|^{2} \geq B^{2}+C^{2}-2 d B C \geq\left(1-d^{2}\right) \max \left\{B^{2}, C^{2}\right\} .
$$

Observe that (2.1) implies that either $\|w\|^{2} \geq \beta_{n-1}^{2} / 2$ or $z^{2} \geq \beta_{n}^{2} / 2$.

In the former case we get

$$
\|P x\|^{2} \geq\left(1-d^{2}\right)\|w\|^{2}\left(1-\|v(n-1)\|^{2}\right) \geq \frac{1-d^{2}}{2} \beta_{n-1}^{2}\left(1-\|v(n-1)\|^{2}\right),
$$

and we are done if we assume that the sequences $\left\{\beta_{n}\right\}$ and $\left\{a_{n}\right\}$ are selected in such a way that

$$
\beta_{n-1}^{2}\left(1-\|v(n-1)\|^{2}\right) \geq \beta_{n}^{2} .
$$

In the latter case we have

$$
\|P x\|^{2} \geq\left(1-d^{2}\right) z^{2}\left(1-a_{n}^{2}\right) \geq \frac{1-d^{2}}{2} \beta_{n}^{2}\left(1-a_{n}^{2}\right) \geq \frac{1-d^{2}}{4} \beta_{n}^{2},
$$

if we assume that $a_{n}^{2} \leq \frac{1}{2}$ for all $n$.

It remains to establish (2.2). Analysis of this inequality shows that we need to prove the inequality

$$
\|v(n-1)\| a_{n} \leq d \sqrt{\left(1-\|v(n-1)\|^{2}\right)\left(1-a_{n}^{2}\right)},
$$

but this follows from the obvious inequality $\|v(n-1)\|^{2} \leq\left(1-a_{n}^{2}\right)$ and the observation that we can select $\left\{a_{n}\right\}$ in such a way that

$$
a_{n}^{2} \leq d^{2}\left(1-\|v(n-1)\|^{2}\right)
$$


This can be done because $1-\|v(n-1)\|^{2}=a_{n}^{2}+a_{n+1}^{2}+\cdots$, and we may select the sequence $\left\{a_{n}\right\}$ in such a way that, for example, $a_{n+1}^{2}+\cdots \geq a_{n}^{2}$.

For clarity we describe the way in which $\left\{a_{i}\right\}$ and $\left\{b_{i}\right\}$ can be chosen. First we choose $\left\{a_{i}\right\}$ in such a way that $\sum_{n} a_{n}^{2}=1, a_{n}^{2} \leq a_{n+1}^{2}+a_{n+2}^{2}+\cdots$, and $a_{n}^{2} \leq \frac{1}{2}$ for all $n$; one of the possible choices is $a_{n}=(\sqrt{2})^{-n}$.

Now we choose $\left\{\beta_{i}\right\}$ satisfying $\beta_{i} \geq \beta_{i+1}>0, \quad \liminf _{n \rightarrow \infty}\left(\beta_{n+1} / \beta_{n}\right)=0$, and (2.3). It is clear that such choice is possible and that with these choices all steps of our argument work.

\section{Dependence of widths on the ambient Banach space}

PROOF OF THEOREM 1.5. It is well known and is easy to see that the unit ball $B_{1}^{3 n}$ of $\ell_{1}^{3 n}$ contains $(1 / \sqrt{3 n}) B_{2}^{3 n}$, where $B_{2}^{3 n}$ is the unit ball of $\ell_{2}^{3 n}$. When we use the term 'orthogonal projection' we mean a projection orthogonal with respect to the inner product corresponding to $\ell_{2}^{3 n}$.

The celebrated result of Kashin [4] (see also [13] and [12, Ch. 6]) states that there is an absolute constant $A$ and a $(2 n)$-dimensional subspace $\mathcal{Y}_{2 n}$ of $\ell_{1}^{3 n}$ such that $\mathcal{Y}_{2 n} \cap B_{1}^{3 n} \subset(A / \sqrt{3 n}) B_{2}^{3 n}$, so that $\mathcal{Y}_{2 n}$ is $A$-isomorphic to a Hilbert space.

We let $P_{n}$ be an orthogonal projection onto $\mathcal{Y}_{2 n}$ and $K_{n}=P_{n}\left(B_{1}^{3 n}\right)$. Since the kernel of $P_{n}$ is $n$-dimensional, it follows that $d_{n}\left(K_{n}, \ell_{1}^{3 n}\right) \leq 1$.

It remains to show that $d_{n}\left(K_{n}, \mathcal{Y}_{2 n}\right) \geq c \sqrt{n}$. Since the norm of $\mathcal{Y}_{2 n}$ is $A$-equivalent to the $\sqrt{3 n}$-multiple of the norm of $\ell_{2}^{3 n}$ restricted to $\mathcal{Y}_{2 n}$, it suffices to show the inequality $d_{n}\left(K_{n}, \mathcal{Y}_{2 n}\right) \geq c_{1}$ with respect to the norm of $\ell_{2}^{3 n}$ on $\mathcal{Y}_{2 n}$.

Let $E_{n}$ be any $n$-dimensional subspace of $\mathcal{Y}_{2 n}$. Let $Q: \mathcal{Y}_{2 n} \rightarrow E_{n}^{\perp}$ be an orthogonal projection onto the orthogonal complement of $E_{n}$ in $\mathcal{Y}_{2 n}$. It suffices to show that the diameter of $Q\left(K_{n}\right)$ with respect to the norm of $\ell_{2}^{3 n}$ is greater than or equal to $c_{1}$.

Let $\left\{e_{j}\right\}_{j=1}^{3 n}$ be the unit vector basis of $\ell_{1}^{3 n}$. We need to show that $\left\|Q P_{n} e_{j}\right\|_{\ell_{2}^{3 n}} \geq c_{1}$ for some $j \in\{1, \ldots, 3 n\}$.

Let $\left\{f_{i}\right\}_{i=1}^{n}$ be an orthonormal basis of $E_{n}^{\perp}$. Then

$$
f_{i}=\sum_{j=1}^{3 n}\left\langle f_{i}, e_{j}\right\rangle e_{j}
$$

Therefore $\sum_{j=1}^{3 n}\left\langle f_{i}, e_{j}\right\rangle^{2}=1$ and

$$
\sum_{i=1}^{n} \sum_{j=1}^{3 n}\left\langle f_{i}, e_{j}\right\rangle^{2}=n
$$

This implies that there exists $j \in\{1,2, \ldots, 3 n\}$ such that

$$
\sum_{i=1}^{n}\left\langle f_{i}, e_{j}\right\rangle^{2} \geq \frac{1}{3},
$$

and this inequality means that $\left\|Q P_{n} e_{j}\right\| \geq 1 / \sqrt{3}$. 
REMARK 3.1. Later we shall need a slight generalization of the statement proved above: the set $K_{n}$ contains $3 n$ vectors $\left\{P_{n} e_{i}\right\}_{i=1}^{3 n}$ such that for each orthogonal projection $Q$ with rank at least $\alpha n(0<\alpha<1)$ on $\mathcal{Y}_{2 n}$ endowed with the norm whose unit ball is $(1 / \sqrt{3 n}) B_{2}^{3 n} \cap \mathcal{Y}_{2 n}$ there exists $j \in\{1, \ldots, 3 n\}$ such that $\left\|Q P_{n} e_{j}\right\| \geq$ $\sqrt{\alpha n}$.

\section{Absolute widths}

We start by mentioning the fact observed in [2, Section 2] that there exists a wide class of compacts $K$ for which their widths in $\overline{\operatorname{lin}(K)}$ is the same as their absolute widths. Examples can be constructed in the following way. Let $\mathcal{Y}$ be an arbitrary infinite-dimensional Banach space. Let $\left\{\mathcal{Z}_{n}\right\}_{n=1}^{\infty}$ be a family of subspaces of $\mathcal{Y}$ satisfying $\operatorname{dim} \mathcal{Z}_{n}=n$ and $\mathcal{Z}_{n} \subset \mathcal{Z}_{n+1}$, let $B_{n}$ be the unit ball of $\mathcal{Z}_{n}$ and let $\left\{t_{n}\right\}$ be a decreasing sequence of positive numbers with $\lim _{n \rightarrow \infty} t_{n}=0$. Consider the compact

$$
K=\overline{\operatorname{conv}\left(\bigcup_{n=1}^{\infty} t_{n} B_{n}\right)} .
$$

Then $d_{n}(K, \mathcal{X})=t_{n+1}$ for each $n \in \mathbb{N}$ and each Banach space $\mathcal{X}$ containing $\mathcal{Y}$ as a subspace. The estimate from above follows from $K \subset \mathcal{Z}_{n}+t_{n+1} B_{\mathcal{X}}$. The estimate from below is an immediate consequence of [7, Theorem 5.1, p. 419].

The proof of Theorem 1.5 can be used to construct an infinite-dimensional compact $K$ in a Hilbert space $\mathcal{H}$ for which $\lim \inf _{n \rightarrow \infty} d_{n}^{a}(K) / d_{n}(K, \mathcal{H})=0$.

See [3, p. 5] for definitions of direct sums used below.

THEOREM 4.1. There exist a compact $K \subset \mathcal{H}$ and an isometric embedding $I$ of $\mathcal{H}$ into a Banach space $\mathcal{X}$ isomorphic to $\mathcal{L}=\left(\sum_{n=1}^{\infty} \ell_{1}^{3 n}\right)_{2}$, such that

$$
\liminf _{n \rightarrow \infty}\left(d_{n}(I K, \mathcal{X}) / d_{n}(K, \mathcal{H})\right)=0 .
$$

PROOF. We let $\left\{n_{i}\right\}_{i=1}^{\infty}$ be an increasing sequence of positive integers satisfying

$$
\sum_{i=1}^{k}\left(2 n_{i}\right) \leq \frac{n_{k+1}}{2} .
$$

We represent $\mathcal{H}$ as $\left(\sum_{i=1}^{\infty} \ell_{2}^{2 n_{i}}\right)_{2}$. We define $E_{i}: \ell_{2}^{2 n_{i}} \rightarrow \ell_{1}^{3 n_{i}}$ as a linear embedding which maps $B_{2}^{2 n_{i}}$ onto the intersection $\left(1 / \sqrt{3 n_{i}}\right) B_{2}^{3 n_{i}} \cap \mathcal{Y}_{2 n_{i}}$, where $\mathcal{Y}_{2 n_{i}}$ are the same as in Theorem 1.5. Combining these embeddings we get an embedding $E$ of $\mathcal{H}$ into $\mathcal{L}$. This embedding is not isometric but it satisfies $\|x\| / A \leq\|E x\| \leq\|x\|$ for the absolute constant $A$ introduced in the proof of Theorem 1.5.

The well-known argument [10, Proposition 1] implies that to complete the proof of the theorem it suffices to find a compact $K \subset \mathcal{H}$ such that

$$
\liminf _{n \rightarrow \infty}\left(d_{n}(E K, \mathcal{L}) / d_{n}(K, \mathcal{H})\right)=0
$$


We let $K_{n_{i}} \subset \mathcal{Y}_{2 n_{i}}$ be the compacts $P_{n_{i}}\left(B_{1}^{3 n_{i}}\right)$ defined in Theorem 1.5 and $H_{i}=$ $E_{i}^{-1} K_{n_{i}}$ be their pre-images considered as compacts in $\ell_{2}^{2 n_{i}}$. The desired compact $K$ will be found in the form $K=\overline{\operatorname{conv}\left(\bigcup_{i=1}^{\infty} \alpha_{i} H_{i}\right)}$, where $H_{i}$ is considered as a subset of $\mathcal{H}$ and $\left\{\alpha_{i}\right\}$ is a converging to zero sequence of positive real numbers satisfying

$$
\alpha_{n} \geq \sum_{i=n+1}^{\infty}\left(\alpha_{i} \operatorname{diam}\left(H_{i}\right)\right) .
$$

We let $p=p_{k}=\sum_{i=1}^{k}\left(2 n_{i}\right)+n_{k+1}$. To complete the proof of the theorem it suffices to show that:

(1) $d_{p}(E K, \mathcal{L}) \leq 2 \alpha_{k+1}$;

(2) $d_{p}(K, \mathcal{H}) \geq \frac{1}{\sqrt{2}} \sqrt{n_{k+1}} \alpha_{k+1}$.

To prove (1) we consider the $p$-dimensional space $\mathcal{L}_{p}=\sum_{n=1}^{k} \mathcal{Y}_{2 n_{i}}+\operatorname{ker} P_{n_{k+1}}$ in $\mathcal{L}$. Then

$$
K \subset \mathcal{L}_{p}+\alpha_{k+1} B_{1}^{3 n_{k+1}}+\overline{\operatorname{conv}\left(\bigcup_{i=k+2}^{\infty} \alpha_{i} K_{n_{i}}\right)} .
$$

Therefore

$$
d_{p}(K, \mathcal{L}) \leq \alpha_{k+1}+\sum_{i=k+2}^{\infty}\left(\alpha_{i} \operatorname{diam}\left(H_{i}\right)\right) \leq 2 \alpha_{k+1}
$$

To prove (2) assume the contrary. Then for some $k$ there is a $p_{k}$-dimensional subspace $\mathcal{A}_{p} \subset \mathcal{H}$ such that $K \subset \mathcal{A}_{p}+c \sqrt{n_{k+1}} \alpha_{k+1} B_{\mathcal{H}}$, where $c<\frac{1}{\sqrt{2}}$. Therefore

$$
\alpha_{k+1} H_{k+1} \subset \mathcal{A}_{p}+c \sqrt{n_{k+1}} \alpha_{k+1} B_{\mathcal{H}}
$$

and

$$
\alpha_{k+1} H_{k+1} \subset \mathcal{Z}_{p}+c \sqrt{n_{k+1}} \alpha_{k+1} B_{\mathcal{H}},
$$

where $\mathcal{Z}_{p}$ is a $p$-dimensional subspace in $\ell_{2}^{2 n_{k+1}}$.

Let $Q: \ell_{2}^{2 n_{k+1}} \rightarrow \mathcal{Z}_{p}^{\perp}$ be an orthogonal projection onto the orthogonal complement of $\mathcal{Z}_{p}$ in $\ell_{2}^{2 n_{k+1}}$. The inequality (4.2) implies that $\operatorname{dim} \mathcal{Z}_{p}^{\perp}=2 n_{k+1}-p \geq \frac{1}{2} n_{k+1}$. By Remark 3.1 there is a vector $z \in \alpha_{k+1} H_{k+1}$ such that $\|Q z\| \geq \frac{1}{\sqrt{2}} \alpha_{k+1} \sqrt{n_{k+1}}$. We arrive at a contradiction.

In connection with the examples presented in this section we suggest the following problems.

Problem 4.2. Does there exist an infinite-dimensional compact $K$ such that

$$
\lim _{n \rightarrow \infty} d_{n}^{a}(K) / d_{n}(K, \overline{\operatorname{lin}(K)})=0 ?
$$

Problem 4.3. Characterize compacts $K$ for which the absolute widths do not differ much from the widths of $K$ in $\overline{\operatorname{lin}(K)}$. 


\section{Acknowledgement}

The author thanks V. S. Shulman for his interest in this work.

\section{References}

[1] C. V. Hutton, 'On the approximation numbers of an operator and its adjoint', Math. Ann. 210 (1974), 277-280.

[2] R. S. Ismagilov, 'Diameters of sets in normed linear spaces, and the approximation of functions by trigonometric polynomials', Uspekhi Mat. Nauk 29(3(177)) (1974), 161-178 (in Russian); Russian Math. Surveys 29(3) (1974), 169-186 (English translation).

[3] W. B. Johnson and J. Lindenstrauss, 'Basic concepts in the geometry of Banach spaces', in: Handbook of the Geometry of Banach Spaces, Vol. 1 (eds. W. B. Johnson and J. Lindenstrauss) (Elsevier, Amsterdam, 2001), pp. 1-84.

[4] B. S. Kashin, 'The widths of certain finite-dimensional sets and classes of smooth functions', Izv. Akad. Nauk SSSR Ser. Mat. 41(2) (1977), 334-351 (in Russian); Math. USSR-Izv. 11(2) (1977), 317-333 (1978) (English translation).

[5] A. S. Kochurov, 'Absolute widths and cowidths', Mat. Zametki 48(1) (1990), 38-46 (in Russian); Math. Notes 48 (1990), 647-652 (English translation).

[6] A. Kolmogoroff, 'Über die beste Annäherung von Funktionen einer gegebenen Funktionenklasse', Ann. of Math. (2) 37 (1936), 107-111.

[7] G. G. Lorentz, M. v. Golitschek and Y. Makovoz, Constructive Approximation. Advanced Problems, Grundlehren der mathematischen Wissenschaften, 304 (Springer, Berlin, 1996).

[8] T. Oikhberg, 'Absolute widths of some embeddings', J. Approx. Theory 81(1) (1995), 120-126.

[9] M. I. Ostrovskii and V. S. Shulman, 'Weak operator topology, operator ranges and operator equations via Kolmogorov widths', Integral Equations Operator Theory 65 (2009), 551-572.

[10] A. Pełczyński, 'Projections in certain Banach spaces', Studia Math. 19 (1960), 209-228.

[11] A. Pinkus, $n$-Widths in the Approximation Theory (Springer, Berlin, 1985).

[12] G. Pisier, The Volume of Convex Bodies and Banach Space Geometry, Cambridge Tracts in Mathematics, 94 (Cambridge University Press, Cambridge, 1989).

[13] S. J. Szarek, 'On Kashin's almost Euclidean orthogonal decomposition of $\ell_{n}^{1}$ ', Bull. Acad. Polon. Sci. Sér. Sci. Math. Astronom. Phys. 26(8) (1978), 691-694.

[14] V. M. Tikhomirov, 'Diameters of sets in functional spaces and the theory of best approximations', Uspekhi Mat. Nauk 15(3) (1960), 81-120 (in Russian); Russian Math. Surveys, 15(3) (1960), 75-111 (English translation).

MIKHAIL I. OSTROVSKII, Department of Mathematics and Computer Science, St. John's University, 8000 Utopia Parkway, Queens, NY 11439, USA e-mail: ostrovsm@stjohns.edu 